\title{
ESCO2 promotes lung adenocarcinoma progression by regulating hnRNPA1 acetylation
}

\author{
Hui-er Zhu' ${ }^{1+}$, Tao Li ${ }^{2+}$, Shengnan Shi ${ }^{3+}$, De-xiong Chen ${ }^{1}$, Weiping Chen ${ }^{4^{*}}$ and Hui Chen ${ }^{5^{*}}$ (D)
}

\begin{abstract}
Background: Emerging evidence indicates that metabolism reprogramming and abnormal acetylation modification play an important role in lung adenocarcinoma (LUAD) progression, although the mechanism is largely unknown.

Methods: Here, we used three public databases (Oncomine, Gene Expression Omnibus [GEO], The Cancer Genome Atlas [TCGA]) to analyze ESCO2 (establishment of cohesion 1 homolog 2) expression in LUAD. The biological function of ESCO2 was studiedusing cell proliferation, colony formation, cell migration, and invasion assays in vitro, and mouse xenograft models in vivo. ESCO2 interacting proteins were searched using gene set enrichment analysis (GSEA) and mass spectrometry. Pyruvate kinase M1/2 (PKM) mRNA splicing assay was performed using RT-PCR together with restriction digestion. LUAD cell metabolism was studied using glucose uptake assays and lactate production. ESCO2 expression was significantly upregulated in LUAD tissues, and higher ESCO2 expression indicated worse prognosis for patients with LUAD.
\end{abstract}

Results: We found that ESCO2 promoted LUAD cell proliferation and metastasis metabolic reprogramming in vitro and in vivo. Mechanistically, ESCO2 increased hnRNPA1 (heterogeneous nuclear ribonucleoprotein A1) binding to the intronic sequences flanking exon 9 (EI9) of PKM mRNA by inhibiting hnRNPA1 nuclear translocation, eventually inhibiting PKM1 isoform formation and inducing PKM2 isoform formation.

Conclusions: Our findings confirm that ESCO2 is a key factor in promoting LUAD malignant progression and suggest that it is a new target for treating LUAD.

Keywords: Acetylation, Metabolism reprogramming, Lung adenocarcinoma, ESCO2, hnRNPA1

\section{Background}

Lung cancer is a heterogeneous tumor with high morbidity and mortality, and is a serious threat to human health $[1,2]$. Lung adenocarcinoma (LUAD) is the most common histologic type of lung cancer, accounting for

\footnotetext{
*Correspondence: 1806973726@qq.com; chenhui7320@126.com

${ }^{\dagger}$ Hui-er Zhu, Tao Li and Shengnan Shi contributed equally to this work.

${ }^{4}$ Department of Respiratory, The People's Hospital of Qingyuan, Sixth Affiliate Hospital of Guangzhou Medical University, Qingyuan 511518, P. R. China ${ }^{5}$ Department of Pathology, The Third Affiliated Hospital of Guangzhou Medical University, Guangzhou Medical University, Guangzhou, Guangdong 510150, P.R. China

Full list of author information is available at the end of the article
}

about $50 \%$ of all lung cancers. On average, more than 7500 people die from cancer every day [3]. More than $35 \%$ of all cancer deaths are from lung cancer [4]. In recent years, many targeted therapies, such as anaplastic lymphoma kinase (ALK) [5], EGFR [6], ROS1 [7], RET [8], HER2 [9], and MEK [10], have become available for advanced lung cancer, and more are in development [11]. Although there are many means of treating lung cancer, no specific drugs have been found so far [12]. Due to tumor heterogeneity, there is an urgent need to identify new therapeutic targets.

(c) The Author(s). 2021 Open Access This article is licensed under a Creative Commons Attribution 4.0 International License, which permits use, sharing, adaptation, distribution and reproduction in any medium or format, as long as you give appropriate credit to the original author(s) and the source, provide a link to the Creative Commons licence, and indicate if changes were made. The images or other third party material in this article are included in the article's Creative Commons licence, unless indicated otherwise in a credit line to the material. If material is not included in the article's Creative Commons licence and your intended use is not permitted by statutory regulation or exceeds the permitted use, you will need to obtain permission directly from the copyright holder. To view a copy of this licence, visit http://creativecommons.org/licenses/by/4.0/ The Creative Commons Public Domain Dedication waiver (http://creativecommons.org/publicdomain/zero/1.0/) applies to the data made available in this article, unless otherwise stated in a credit line to the data. 
Establishment of cohesion 1 homolog 2 (ESCO2) is an evolutionarily conserved cohesion acetyltransferase that exerts essential functions in the establishment of sister chromatid cohesion [13]. In recent years, ESCO2 has been identified as an essential factor in cancer progression in multiple human cancers [14-17]. Compared with conventional chemotherapy, ESCO2 may become a more promising option for renal cell carcinoma treatment intervention [15]. ESCO2 is highly expressed in aggressive melanomas and breast cancer $[16,18]$. In gastric cancer, ESCO2 promotes cell proliferation by modulating the p53 and mammalian target of rapamycin (mTOR) signaling pathways [19]. However, its biological function and clinical significance in lung cancer remain unclear.

Heterogeneous nuclear ribonucleoprotein A1 (hnRNPA1) is an RNA-binding protein that associates with pre-mRNAs in the nucleus and influences premRNA processing, as well as other aspects of mRNA metabolism and transport, and plays a key role in regulating alternative splicing $[20,21]$. The pyruvate kinaseisoform PKM2 is widely expressed in cancer for maintaining glycolysis-dominant energy metabolism, while PKM1 promotes oxidative phosphorylation [22]. These two isoforms result from mutually exclusive alternative splicing of $P K M$ pre-mRNA, reflecting the inclusion of either exon 9 (PKM1) or exon 10 (PKM2), required for tumor cell proliferation [23]. Clinical cancer samples support the notion that hnRNPA1 overexpression decreases the PKM1/PKM2 ratio, which has a positive effect on glycolysis-dominant metabolism [24].

In the present research, we found that high ESCO2 expression in LUAD was associated with poor prognosis. Overexpression of ESCO2 promoted LUAD cell proliferation, colony formation, migration, and invasion in vitro, while ESCO2 knockdown inhibited LUAD cell malignant progression in vitro and tumorigenesis and metastasis in vivo. Coimmunoprecipitation (Co-IP) and mass spectrometry (MS) analysis suggested that ESCO2 could interact with hnRNPA1, which is involved in mRNA splicing or processing. Moreover, we found that ESCO2 can acetylate hnRNPA1 at lysine 277 (K277) to retain hnRNPA1 in the nucleus. Only in the nucleus can hnRNPA1 regulate PKM splicing to promote PKM2 generation and inhibit PKM1 generation, leading to LUAD metabolism reprogramming. The present study indicates the functional roles of ESCO2 in LUAD progression and that ESCO2 may be a potential therapeutic target for LUAD.

\section{Materials and methods}

\section{Tissue samples and cell culture}

Primary cancer tissue and normal lung tissue were collected from patients with lung cancer at the
SixthAffiliated Hospital of Guangzhou Medical University. The cases were collected based on a clear pathological diagnosis and patient consent, and the study was approved by the Internal Review and Science Committee of the Sixth Affiliated Hospital of Guangzhou Medical University. The human LUAD cell lines A549 and NCIH1975 were purchased from the Cell Bank of the Chinese Academy of Sciences (Shanghai, China) and maintained in RPMI 1640 medium supplemented with 10\% fetal bovine serum (FBS). HEK293T cells were purchased from ATCC and cultured in Dulbecco's modified Eagle's medium (DMEM) containing 10\% FBS. All cells were maintained at $37^{\circ} \mathrm{C}$ and $5 \% \mathrm{CO}_{2}$ in a humidified incubator.

\section{Public database analysis and gene set enrichment} analysis (GSEA)

LUAD gene expression datasets of Garber et al., Hou et al. and Okamaya et al. were analyzed via Oncomine database (https://www.oncomine.org). LUAD gene expression datasets (GSE74706, GSE21933, GSE32863, GSE50081 and 31,210) were downloaded from the Gene Expression Omnibus (http://www.ncbi.nlm.nih.gov/geo) database. 515 LUAD and 59 normal lung tissue samples were obtained from The Cancer Genome Atlas (TCGA) dataset (https://portal.gdc.cancer.gov/). The TCGA LUADsamples were subdivided into high and low ESCO2expression groups and analyzed with GSEA 2.0.9 software (http://www.broadinstitute.org/gsea/).

\section{Plasmid constructs, transfection, and stable silencing}

Plasmids were constructed by homologous recombination. Briefly, after primer design and synthesis, complementary DNA (cDNA) was amplified using Phanta Max Super-Fidelity DNA Polymerase (Vazyme, cat: C505). The PCR products were purified and recovered according to the protocol of a general DNA purification and recovery kit (Tiangen Biochemical Technology, DP214-03). Then, recombination, transformation, coating, cloning identification, and plasmid extraction were performed. The mutants of hnRNP A1-HA were produced usingMut Express II Fast Mutagenesis Kit V2 (vazyme, C214). The A549 and NCI-H1975 cells were transfected with overexpression vector using $\mathrm{Li}$ pofectamine 2000 (Thermo Fisher Scientific, MA, USA). LUAD cell lines with stable ESCO2 silencing were constructed using lentivirus pLV3short hairpin RNA (shRNA) as previously described [4]. The lentivirus pLV3-ESCO2 shRNA was purchased from GenePharma (Shanghai, China). The sequences of the primers and shRNAs used in the study are listed in Supplementary Table S1. 


\section{Immunofluorescence staining}

NCI-H1975 cells were transfected with ESCO2-FLAG vectors, and plated on glass coverslips. The cell density was about 50\%; the cells were rinsed with phosphatebuffered saline (PBS) twice, fixed with $1 \mathrm{~mL} \mathrm{4 \%} \mathrm{parafor-}$ maldehyde at room temperature for $20 \mathrm{~min}$, and permeabilized with $0.1 \%$ Triton X-100 for $7 \mathrm{~min}$. The cells were rinsed twice with precooled PBS and blocked with 2\% BSA (bovine serum albumin) at room temperature for $2 \mathrm{~h}$. Primary antibody was added and incubated at $4{ }^{\circ} \mathrm{C}$ overnight, following which the samples were incubated with secondary antibodies. The nuclei were stained with DAPI, and examined under a microscope.

\section{Quantitative real-time PCR (RT-qPCR)}

Total RNA was extracted from treated A549 and NCIH1975 cells using TRIzol total RNA isolation reagent (Invitrogen). Then, cDNA was synthesized from the total RNA using a PrimeScript RT Reagent Kit (TAKARA). ESCO2 mRNA expression was detected using quantitative PCR (q-PCR) following the manufacturer's protocol. ESCO2 and GAPDH expression levels were measured using the comparative threshold cycle (2- $\Delta \Delta \mathrm{Ct})$ method. The primer sequences used are listed in Supplementary Table S1.

\section{Western blotting}

Proteins were extracted from cells or tissue using lysis buffer (1 mM EDTA, 1\% SDS, $5 \mathrm{mM}$ DTT, $10 \mathrm{mM}$ PMSF, 50 mMTris-HCl [pH 8.0], protease inhibitor cocktail). Protein concentrations were determined using the bicinchoninic acid (BCA) assay. Total cell lysates were fractionated by $8 \%$ or $10 \%$ sodium dodecyl sulfatepolyacrylamide gel electrophoresis (SDS-PAGE), transferred to PVDF membranes. The primary antibodies used are listed in Supplementary Table S1.

\section{Cell growth and colony formation assays}

For the cell growth assay, $1 \times 10^{4}$ treated LUAD cells were seeded in 24-well plates, and counted at 24, 48, 72, 96 , and $120 \mathrm{~h}$. For the colony formation assays, $5 \times 102$ treated LUAD cells were seeded in 6-well plates and cultured in RPMI 1640 medium containing 10\% FBS for 8 or 10 days. The clones were fixed in methanol and stained with crystal violet solution.

\section{Migration and invasion assays}

The in vitro migration and invasion assays were performed using Transwell chambers. For the migration assay, $1 \times 10^{5}$ LUAD cells with ESCO2 overexpression or silencing were cultured in RPMI 1640 medium in the upper compartment of a Transwell chamber. For the invasion assay, $2 \times 10^{5}$ LUAD cells with ESCO2 overexpression were resuspended in RPMI 1640 medium with
0.1\% FBS in Matrigel-coated upper Transwell chambers. For both assays, the bottom chambers were filled with RPMI 1640 medium containing 10\% FBS. The chambers were stained with $0.5 \%$ crystal violet. Migrated and invaded cells were counted under a microscope.

\section{In vivo xenograft tumor model}

All animal procedures were approved by the Institutional Animal Care and Use Committee of the ThirdAffiliated Hospital of Guangzhou Medical University. For the in vivo tumor growth assay, $5 \times 10^{6}$ control or ESCO2 knockdown NCI-H1975 cells were injected into the left and right flanks of BALB/c null mice $(n=6)$. After 21 days, all tumors were stripped and weighed. For the in vivo metastasis assay, control or ESCO2 knockdown NCI-H1975 cells were luciferase (Luc)-labeled using the lentivirus system. NCI-H1975-Luc-NC (negative control) or NCI-H1975-Luc ESCO2shRNA cells $\left(2 \times 10^{6}\right.$ cells $)$ were injected into the tail veins of NODSCID (nonobese diabetic/severe combined immunodeficient) mice. After 45 days, the metastatic foci were detected using the IVIS 200 imaging system (Xenogen, Alameda, CA, USA).

\section{Silver staining and mass spectrometry (MS)}

HEK293T cells were transfected with Flag-ESCO2 vector for $48 \mathrm{~h}$ using Lipofectamine 2000. Treated HEK293T cells were lysed in Co-IP lysis buffer (P0013, Beyotime, Shanghai, China). Co-IP was performed using antiFLAG/anti-HAantibodiesand protein A/G agarose beads (sc-2003, Santa Cruz) to extractthe complexes. Gel bands were detected using a silver staining kit (P0017S, Beyotime) combined with MS following the manufacturer's protocol. According to a previously published method [25], the peptides of the bands were analyzed using nano-LC-MS/MS. The mass spectrometry proteomics data have been deposited to the ProteomeXchange Consortium via the PRIDE partner repository with the dataset identifier PXD023527 and PXD23600.

\section{In vitro acetylation assay}

FLAG-tagged ESCO2, HA-tagged hnRNPA1, and their mutant proteins were purified from HEK293T cells using a FLAG Immunoprecipitation Kit (FLAGIPT1, Sigma) or Anti-HA Immunoprecipitation Kit (IP0010, Sigma). Recombinant ESCO2 proteins were incubated with recombinant hnRNPA1 or its mutants in $30 \mu \mathrm{L}$ reaction buffer ( $50 \mathrm{mMNaCl}, 50 \mathrm{mMTris}-\mathrm{HCl}$ [pH 8.0], 4 $\mathrm{mM} \mathrm{MgCl}_{2}, 1 \mathrm{mM}$ DTT, $0.1 \mathrm{mM}$ EDTA, $10 \%$ glycerol) at $37^{\circ} \mathrm{C}$ for $30 \mathrm{~min}$.

\section{RNA affinity purification}

First, pretreatment streptomycin beads: $100 \mu \mathrm{L}$ streptavidin-agarose beads were rinsed twice using pre- 
cooled $500 \mu$ Lbinding buffer ( $\mathrm{pH} 7.5$ ), centrifuged at $4{ }^{\circ} \mathrm{C}$ at $2500 \mathrm{rpm}$ for $5 \mathrm{~min}$, and the supernatant was discarded. Then, $1 \mathrm{nmol}$ biotin probe-labeled RNA fragments were bound with $100 \mu \mathrm{L}$ streptavidin-agarose beads at $4{ }^{\circ} \mathrm{C}$ overnight. Cellular nuclear protein was prepared using a Nuclear and Cytoplasmic Extraction Kit (Beyotime). Purified protein or nucleoprotein and tRNA were added to the beads, incubated at $30^{\circ} \mathrm{C}$ for $10 \mathrm{~min}$, centrifuged at $4{ }^{\circ} \mathrm{C}$ at $2500 \mathrm{rpm}$ for $5 \mathrm{~min}$, and the supernatant was discarded. Then, the pretreatment protein was added, incubated at $4{ }^{\circ} \mathrm{C}$ for $2 \mathrm{~h}$, centrifuged at $2500 \mathrm{rpm}$ for $5 \mathrm{~min}$ at $4{ }^{\circ} \mathrm{C}$, rinsed twice with precooled binding buffer, and centrifuged at $2500 \mathrm{rpm}$ for 5 min at $4{ }^{\circ} \mathrm{C}$. Finally, the elucidated mixtures were detected using western blotting. The 5' biotin-labeled RNAs used in the study are listed in Supplementary Table S1.

\section{RT-PCR and PKM splicing assays}

PKM splicing assays were performed according to a previous study [26]. Briefly, total mRNA was extracted from cells or tissue samples using TRIzol. mRNA reverse transcription was performed using the PrimeScript RT Reagent Kit (TAKARA). The PCR products were digested using PstI, and the digested mixtures were resolved by $8 \%$ non-denaturing PAGE. The primers used are listed in Supplementary Table S1.

\section{Measurement of glucose uptake and lactate production} A549 and NCI-H1975 cells at the logarithmic growth stage were inoculated in a 12 -well plate at $1 \times 10^{5}$ cells/well. The experiment was divided into the control group (non-transfected cells) and transfection group. After $36 \mathrm{~h}$, the cells were incubated with phenol red-free RPMI 1640 medium for $8 \mathrm{~h}$, and the glucose content in the culture supernatant was detected using Glucose Colorimetric Assay kit (BioVision, K606-100) according to the operating instructions. The glucose content of the non-transfected group was used as the control.

Treated LUAD cells were seeded into 6-well plates. Lactate production was measured using a Lactate Colorimetric Assay Kit II (BioVision, K627-100) according to the manufacturer's protocol. Briefly, at $36 \mathrm{~h}$ posttransfection, phenol red-free RPMI 1640 medium without FBS was added to a 6-well plate of subconfluent cells and cultured for $4 \mathrm{~h}$. A standard curve of nmol/well versus the OD450nm (optical density at $450 \mathrm{~nm}$ ) value was plotted according to the measurement of the lactate standard. The OD450nm values of the sample were applied to the standard curve to calculate the lactate concentrations of the test samples $(n=3)$.

\section{Statistical analysis}

Data are presented as the mean \pm standard deviation (SD); data analysis was performed using GraphPad Prism 5. Survival curves were described using Kaplan-Meier plots and were calculated using the log-rank test. Statistical differences between two groups were analyzed using an independent Student's $t$-test (2-tailed). $P<0.05, p<$ 0.01 , and $p<0.001$ were considered statistically significant.

\section{Results}

ESCO2 is upregulated andassociated with poor prognosis in LUAD

ESCO2 mRNA expression in normal lung tissues and LUAD tissues (Garbar lung, Hou lung, Okamaya lung) was detected using the Oncomine database. We found that ESCO2 expression was significantly higher in LUAD tissues than in normal tissues $(p=0.001, p=3.15 \mathrm{E}-10$ and $p=5.73 \mathrm{E}-8$; Fig. 1a-c). Three LUAD mRNA expression profiling datasets (GSE21933, GSE10072, GSE32863) were analyzed for the ESCO2 mRNA expression levels between LUAD tissues and their adjacent normal tissues. The analysis showed that ESCO2 expression was significantly upregulated in LUAD tissues compared with the adjacent normal lung tissues $(p=0.0004$, $p<0.0001$ and $p=0.0379$; Fig. $1 \mathrm{~d}-\mathrm{f}$ ).

To elucidate the relevance of ESCO2 overexpression to the survival of patients with LUAD, two publicly accessible microarray datasets of patients with LUAD were analyzed. From the GSE50081 and GSE31210 datasets, patients with high $E S C O 2$ expression had shorter overall survival (OS) ( $p=0.0002$ and $p=0.0450$; Fig. $1 \mathrm{~g}$ and $\mathrm{h})$. Moreover, The Cancer Genome Atlas (TCGA) database showed that ESCO2 gene expression levels in LUAD tissue was significantly higher than that in normal lung tissue $(p<0.0001$; Fig. 1i). TCGA LUAD cohort showed that, compared with patients with low ESCO2 expression (the lowest 30\%), patients with high ESCO2 mRNA expression (the highest 30\%) had higher rates of death, shorter disease-free survival, and shorter OS $(p=0.0058$, $p=0.0005$ and $p=0.0013$; Fig. $1 \mathrm{j}-1$ ). We performed a chi-square test to clarify the correlation between ESCO2 low and high expression on the clinicopathological features of LUAD. Our findings indicated that high ESCO2 mRNA expression levels werecorrelated significantly positively with $\mathrm{pT}$ status $(p=0.001), \mathrm{pN}$ status $(p=$ $0.003)$, and clinical stage $(p=0.005)$ (Table 1$)$. To demonstrate ESCO2 expression in LUAD tissues, we performed $\mathrm{qPCR}$ and western blotting on five LUAD tissues and the corresponding non-tumor (NT) tissue samples; the results showed that ESCO2 mRNA and protein expression levels werelower in the NT tissues than in the LUAD tissues (Fig. $1 \mathrm{~m}$ and $\mathrm{n}$ ). Collectively, our findings indicate that ESCO2 level was significantly upregulated 


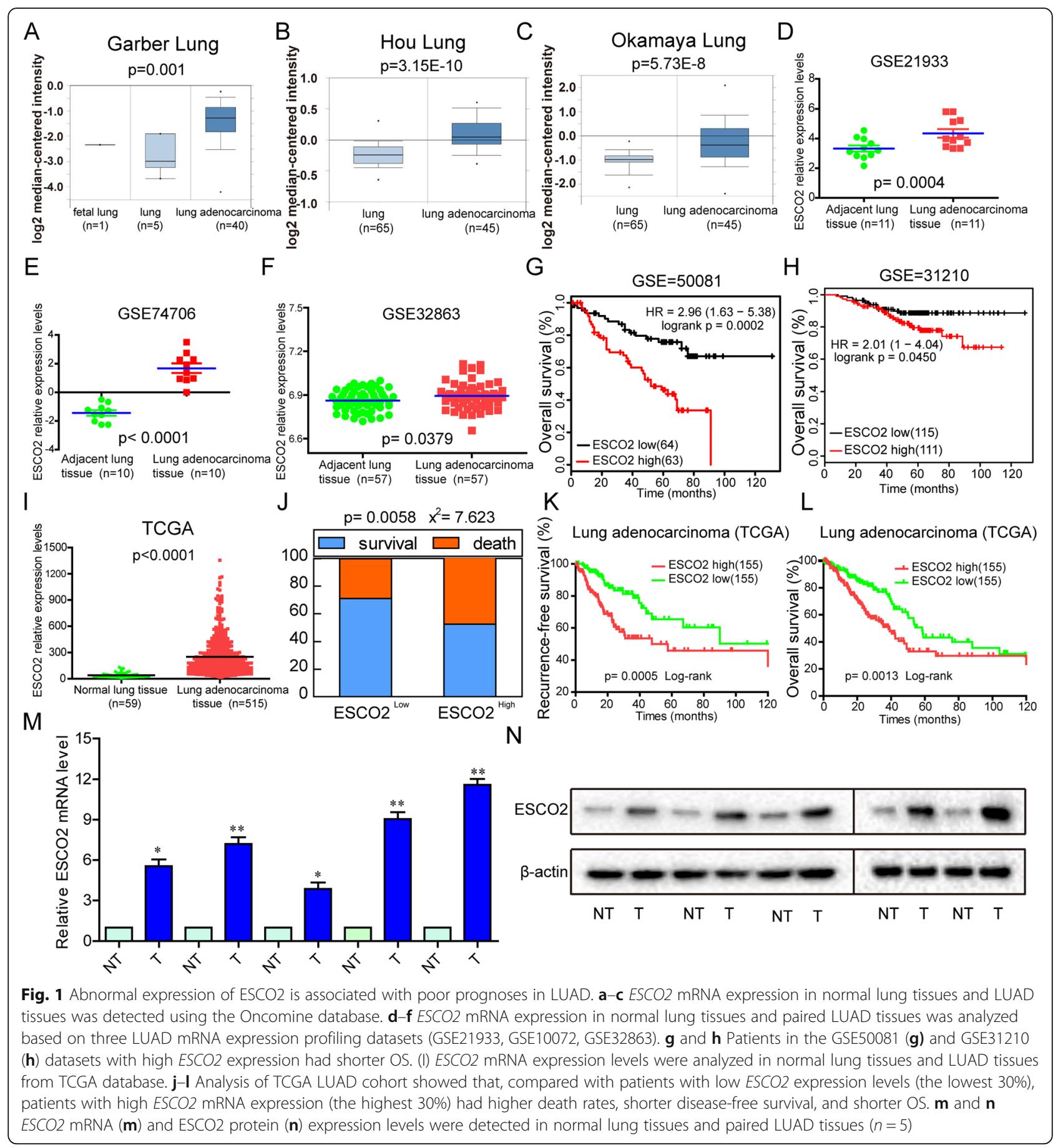

in LUAD and was significantly negatively correlated with OS and DFS, suggesting that ESCO2 may serve as a molecular marker for LUAD treatment and as a promoter of tumorigenesis.

\section{ESCO2 overexpression promotes the malignant phenotype of LUAD cells}

To demonstrate the influence of ESCO2 on cancer growth, metastasis, and colony formation, we generated
aESCO2-FLAG construct, where the FLAG tag (six amino acids) was fused to the full-length $E S C O 2$ transcript. The constructs were transfected into NCI-H1975 and A549 cells, and the expression of the construct was examined using immunofluorescence staining, RT-qPCR, and western blotting. The results suggested the successful transfection of ESCO2 into the cells (Fig. 2a-c).

The global mRNA expression profiles of TCGA LUAD were detected using gene set enrichment analysis 
Table 1 Comparison of clinical features between LUAD patients with low and high ESCO2 levels in TCGA database

\begin{tabular}{|c|c|c|c|c|c|}
\hline \multirow[t]{2}{*}{ Clinical character ${ }^{\#}$} & \multirow{2}{*}{$\begin{array}{l}\text { Clinical } \\
\text { groups }\end{array}$} & \multicolumn{2}{|l|}{ ESCO2 } & \multirow[t]{2}{*}{$x^{2}$} & \multirow[t]{2}{*}{$p$ value } \\
\hline & & $\begin{array}{l}\text { High } \\
(n=155)(\%)\end{array}$ & $\begin{array}{l}\text {-Low } \\
(n=155)(\%)\end{array}$ & & \\
\hline \multirow[t]{2}{*}{ Age (years) } & $\leq 60$ & $54(34.8)$ & $41(26.5)$ & 2.611 & 0.106 \\
\hline & $>60$ & $95(61.3)$ & $108(69.7)$ & & \\
\hline \multirow[t]{2}{*}{ Gender*** } & Male & $91(58.7)$ & $59(38.1)$ & 13.23 & $<0.001$ \\
\hline & Female & $64(41.3)$ & $96(61.9)$ & & \\
\hline \multirow[t]{2}{*}{ ALK Translocation } & No & $54(34.8)$ & $71(45.8)$ & 0.377 & 0.539 \\
\hline & Yes & $12(7.7)$ & $12(7.7)$ & & \\
\hline \multirow[t]{2}{*}{ pT status*** } & $\mathrm{T} 1 \mathrm{~T} 2 \sim \mathrm{T} 4$ & $37(23.9)$ & $66(42.6)$ & 12.27 & $<0.001$ \\
\hline & & $117(75.5)$ & $88(56.8)$ & & \\
\hline \multirow[t]{2}{*}{ pN status** } & No & $88(56.8)$ & $110(71.0)$ & 8.771 & 0.003 \\
\hline & $\mathrm{N} 1 \sim \mathrm{N} 2$ & $66(42.6)$ & $40(25.8)$ & & \\
\hline \multirow[t]{2}{*}{ pM status* } & Mo & $106(68.4)$ & $101(65.2)$ & 4.026 & 0.045 \\
\hline & M1 & $13(8.4)$ & $4(2.6)$ & & \\
\hline \multirow[t]{2}{*}{ Recurred/Progressed** } & No & $62(40.0)$ & $88(56.8)$ & 8.317 & 0.004 \\
\hline & Yes & $67(43.2)$ & $46(29.7)$ & & \\
\hline \multirow[t]{2}{*}{ Clinical Stage ${ }^{* *}$} & Stage I $\sim \| \mathrm{A}$ & $83(53.5)$ & $106(68.4)$ & 7.761 & 0.005 \\
\hline & Stage $\| \mathrm{B} \sim \mathrm{IV}$ & $70(45.2)$ & $46(29.7)$ & & \\
\hline
\end{tabular}

Differences with ${ }^{*} p<0.05,{ }^{* *} p<0.01$ or ${ }^{* * *} p<0.001$ were considered statistically significant

\#American Joint Committee on Cancer classification (Version 7) (AJCC)

(GSEA) software. GSEA plots showed that the cell cycle signatures $(p<0.001)$ and DNA replication signatures $(p=0.007)$ had a significantly positive correlation with ESCO2 mRNA expression levels in TCGA LUAD cohort (Fig. 2d). To identify the effects of ESCO2 overexpression in the malignant phenotype of LUAD cells, the ESCO2 construct was transiently transfected into NCIH1975 and A549 cells. ESCO2 overexpression significantly promoted cell growth in both NCI-H1975 and A549 cells ( $p=0.0011$ and $p=0.0012$; Fig. $2 \mathrm{e}$ ) and significantly increased cell invasion and migration (Fig. 2f). Colony formation was also significantly increased significantly in the ESCO2 overexpression LUAD cells ( $p=$ 0.0054 and $p=0.0051$; Fig. $2 \mathrm{~g}$ ).

\section{Stable silencing of ESCO2 inhibited the aggressive phenotype of LUAD cells in vitro and in vivo}

To prove the influence of ESCO2 on the aggressive phenotypeof LUAD cells in vitro, and on growth and metastasis in vivo, ESCO2 expression was stably silenced using shRNA. ESCO2 mRNA and protein expression levels were detected by RT-qPCR and western blotting, respectively. Comparing with the control shRNA group, the ESCO2 $\operatorname{shRNA}-1(p<0.001)$ and ESCO2 shRNA-2 $(p<0.001)$ groups had significantly decreased ESCO2 mRNA and protein levels (Fig. 3a). Comparing with the control shRNA group, the ESCO2 shRNA-1and ESCO2 shRNA-2 groups had alsoobviouslydecreased ESCO2 protein expression (Fig. 3b). ESCO2 silencing significantly inhibited cell growth (Fig. 3c), migration and invasion (Fig. 3d), and colony formation (Fig. 3e) in both the NCI-H1975 and A549 cells.

In addition, we observed that, contrary to the control shRNA, NCI-H1975 cells with ESCO2 stable silencing had lower tumor volumes and significantly decreased tumor weights ( $p<0.0001$; Fig. 3f). ESCO2 stable silencing also suppressed metastasis ability in vivo (Fig. 3g). At the same time, analysis of the lung metastatic nodule number showed that the ESCO2 shRNA-1 group had significantly decreased metastatic nodules $(p=0.0025$; Fig. 3h).

\section{ESCO2 acetylated hnRNPA1 at K277}

The mechanism of ESCO2 action in LUAD progression was investigated using immunoprecipitation, silver staining, and MS, and 32ESCO2-binding proteins were identified (Fig. 4a and Table S2). The GSEA plot indicated that enrichment of the spliceosome-related genes was significantly correlated to high ESCO2 mRNA expression levels in TCGA LUAD cohort ( $p<0.001$; Fig. 4b and c). The MS showed that hnRNPA1 had a high mass spectrum score (Table S2), and GSEA results and previous study showed that it is an important splicing regulator [27]. According to the results of the MS and GSEA, we found hnRNP A1 to be particularly interesting. To determine the interaction between ESCO2 and 


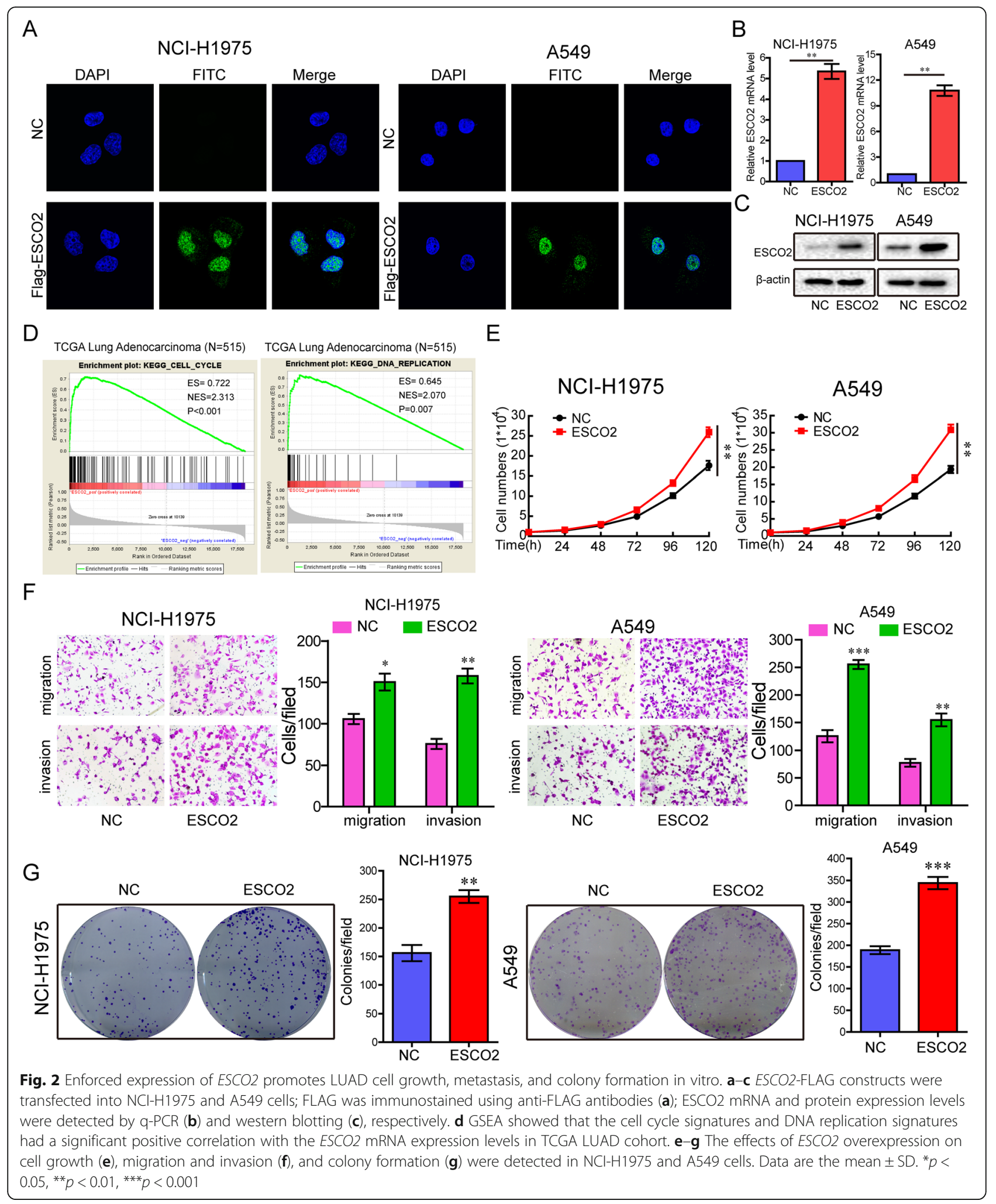

hnRNPA1, the ESCO2-FLAG vector was transfected into NCI-H1975 cells, the ESCO2-FLAG complexes underwent Co-IP, and then hnRNPA1 in the complexes was detected (Fig. 4d). NCI-H1975 cells were transfected with hnRNPA1-HAplasmids, the hnRNPA1-HA complexes underwent Co-IP, and then ESCO2 in the complexes was detected (Fig. 4e). As ESCO2 is an evolutionarily conserved cohesion acetyltransferase, we 


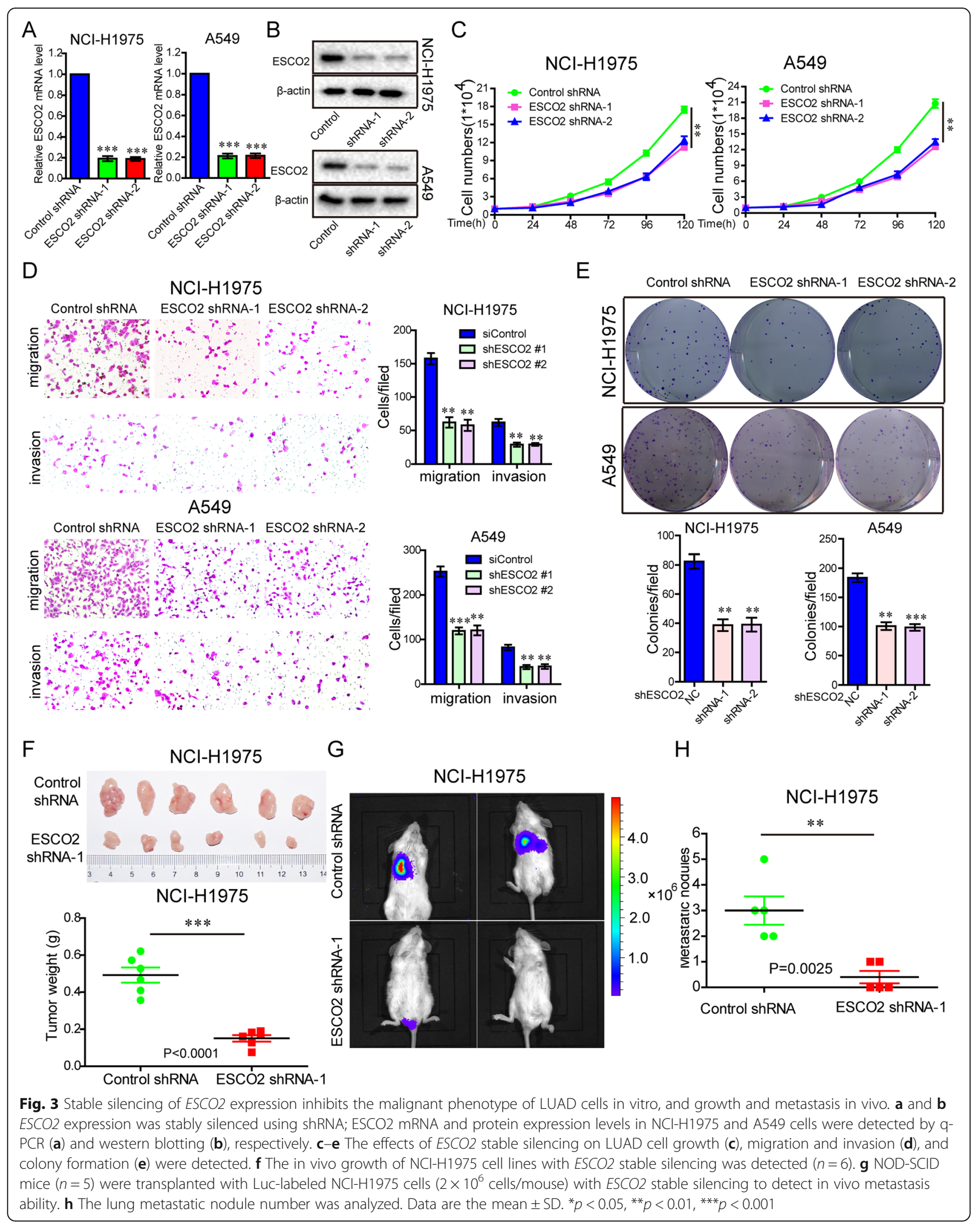


A

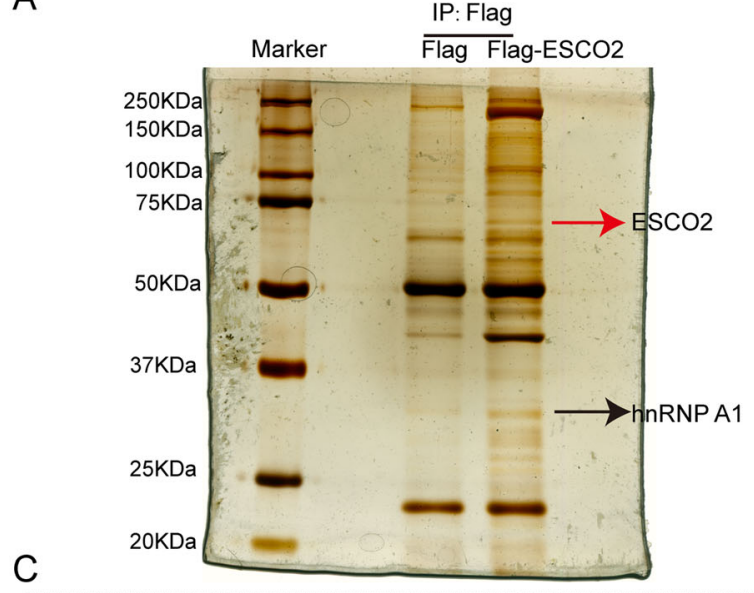

B
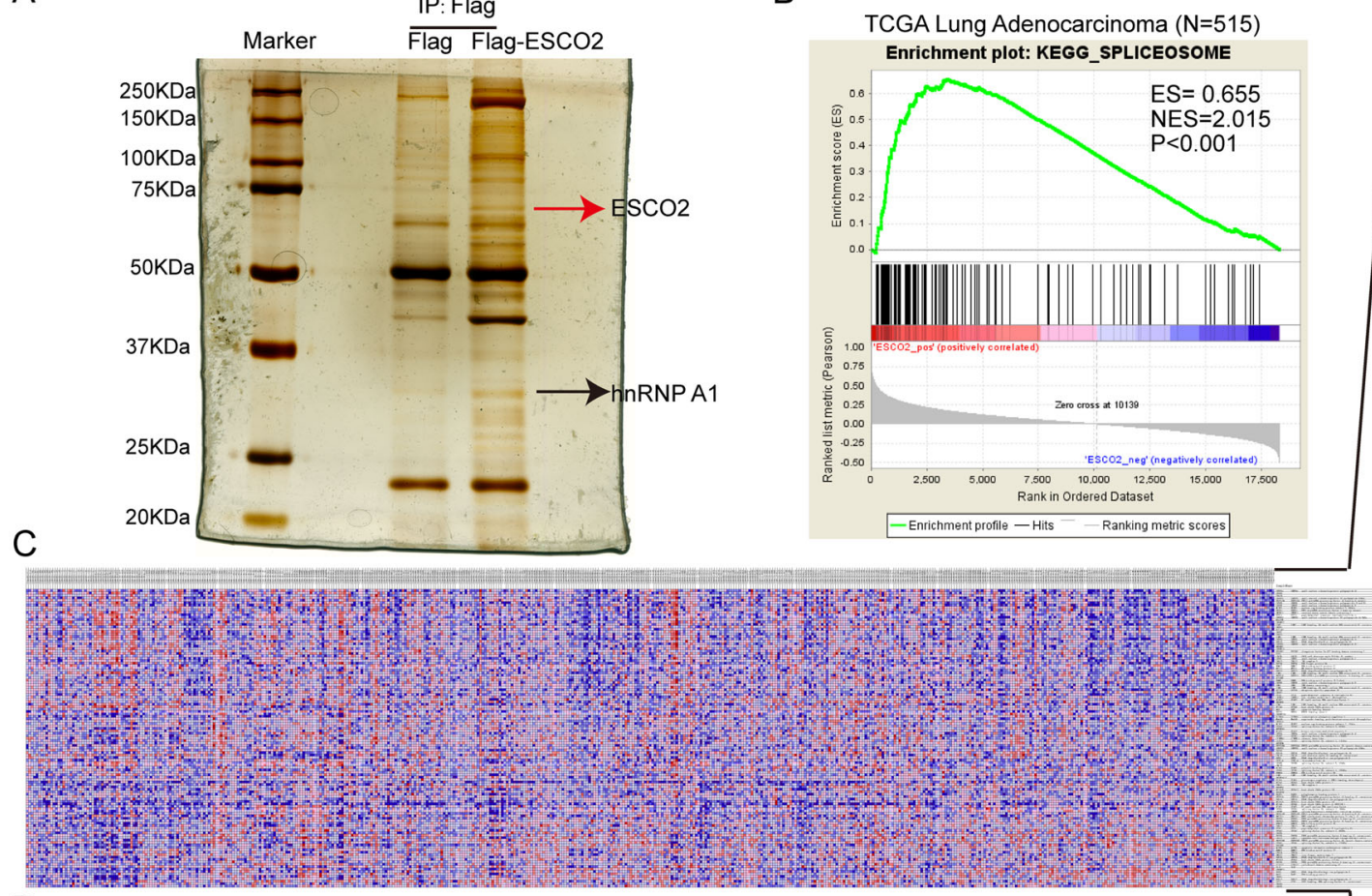

D

IP: Flag $\quad F$

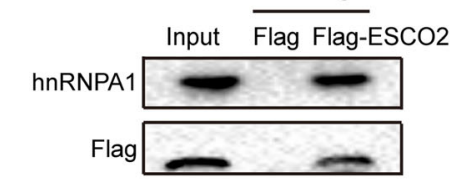

E

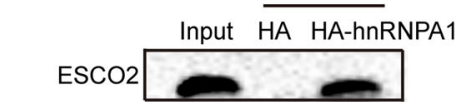

$\mathrm{H}$

$\mathrm{HA}$

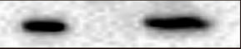

FGNYNNQSSNFGPMK(Ac)GG

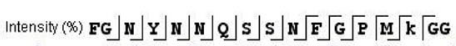

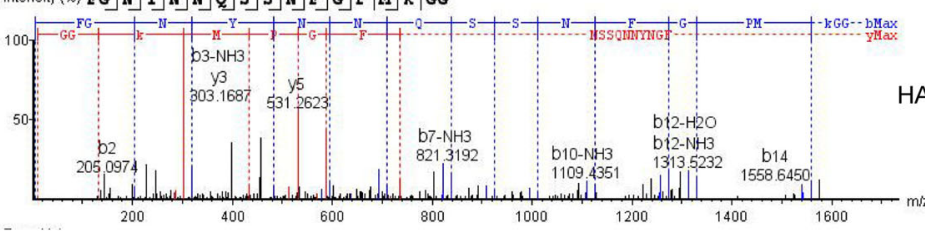

$J$

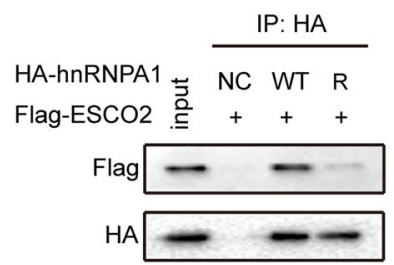

$\mathrm{K}$

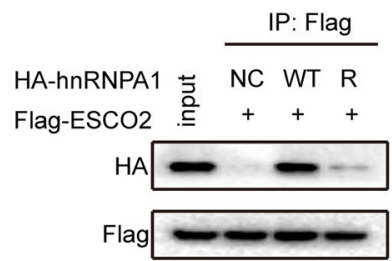

G

ShESCO2 NC sh\#1 sh\#2 HA-hnRNPA1 +++

HA-hnRNPA1 $\longrightarrow$
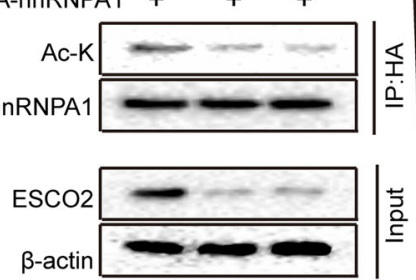

I

ESCO2 - + + HA-hnRNPA1 WT WT K277R

HA-hnRNPA1
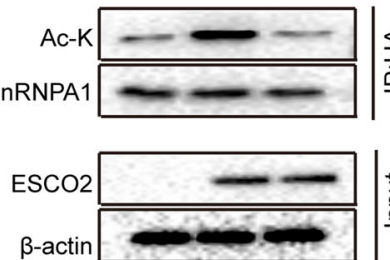

L

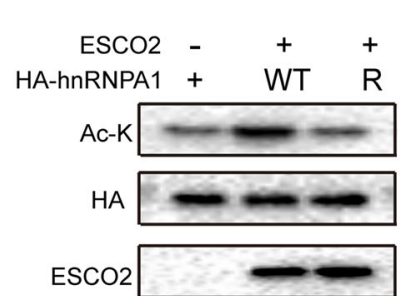

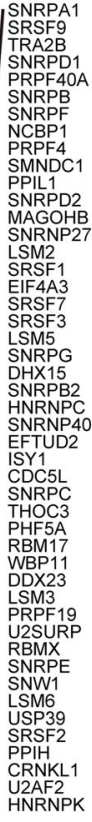

HNRNPA3

BCAS2
SNNPA
SN 3 A

CTNNBL1

SF3B3 3

HNRNPM
PRPFBSA

SNRPD3

$\mathrm{PD} \times 6$
$\mathrm{DDC} 40$
$\mathrm{CD} 40$

THX8
DXNA

TXXN4A

CWCA
CWC15
PCBP1
P

SFFB1
RBMBA

-SMT
INRPA1L2

PLRG1

HSPA2

HNRRPU
HSPA1B
HSA

HNRNPA
POBP1

PRPF18

DHX16
HSPAIA
HSA

MISAG

SE $3 B 4$
CHEP
CHER

PRPF $00 B$
NHPLI1

NHP2L1
PRPE

政

$\mathrm{RBM} 25$
$\mathrm{TH} \mathrm{OC2}$

$P P I E E^{2}$
SFBA2
PBS

SRSE

RBM22

TRA2A
ZMATI
PATS

DHX38
HSPA1
DSP

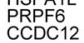

SLU7

DDX5

XAB2

-10
SRSF 4
DDX42
SYF2

SYF2
SRSF8
SRSF5

Fig. 4 (See legend on next page.) 
(See figure on previous page.)

Fig. 4 Interaction of ESCO2 with hnRNPA1 acetylates hnRNPA1 at K277. a ESCO2-FLAG vector was transfected into HEK293T cells, the ESCO2FLAG complexes underwent Co-IP, and then ESCO2-binding proteins were identified by combined silver staining and MS. $\mathbf{b}$ Enrichment plot showing enrichment of spliceosome-related genes in the ESCO2 high-expression group in TCGA LUAD cohort. c Heatmap showing the relative expression values for 126 spliceosome-related genes in TCGA LUAD cohort. d The ESCO2-FLAG vector was transfected into NCI-H1975 cells, the ESCO2-FLAG complexes underwent Co-IP, and then hnRNPA1 in the complexes was detected. e hnRNPA7-HAplasmids were transfected into NClH1975 cells, the hnRNPA1-HA complexes underwent Co-IP, and then ESCO2 in the complexes was detected. $\mathbf{f}$ NCI-H1975 cells were

simultaneously transfected with hnRNPA1-HAand ESCO2-FLAGvectors, immunoprecipitated with anti-HA antibody, and then detection was performed using anti-ac-K antibody. $\mathbf{g}$ NCI-H1975 cells with ESCO2 stable silencing were transfected with hnRNPA1-HA vector,

immunoprecipitated by anti-HA antibody, and then detection was performed using anti-ac-K antibody. $\mathbf{h}$ The K277 acetylation site was identified by MS. i hnRNPA7 WT or K277R mutant plasmids were co-transfected with ESCO2-FLAG into NCI-H1975 cells, and acetylation levels were detected using anti-ac-K antibody. $\mathbf{j}$ and $\mathbf{k}$ hnRNPA1 WT or K277R mutant plasmids were co-transfected with ESCO2-FLAG into NCl-H1975 cells, anti-HA antibody was used for Co-IP, and ESCO2-FLAG was detected using anti-FLAG antibody (j); anti-FLAG antibody was used for Co-IP, and hnRNPA1 WT and K277R mutant constructs were detected using anti-HA antibody (k). Recombinant WT hnRNPA1 and its mutant K277R were incubated with recombinant ESCO2, and acetylation levels were detected using anti-ac-K antibody

examined whether ESCO2 acetylates hnRNPA1 by cotransfecting NCI-H1975 cells with hnRNPA1-HAand ESCO2-FLAGvectors, followed by immunoprecipitation by anti-HA antibody, and detection using the panspecific anti-acetylated lysineantibody. The hnRNPA1 acetylation levels were increased (Fig. 4f), suggesting that ESCO2 can acetylate hnRNPA1 protein. In addition, compared with the NC group, the hnRNPA1 acetylation levels in the shESCO2 group was decreased significantly (Fig. 4g). Co-IP confirmed that there was an interaction between ESCO2 and hnRNPA1 protein and that ESCO2 could acetylate hRNPA1 protein, but the acetylation site was not known. The acetylation site was identified by Co-IP combined with MS (Fig. 4g and Fig. S1), and was revealed to be on lysine $(\mathrm{K})$ at site 277 . To investigate whether the acetylation site is at K277, hnRNPA1 WT (wild-type) or K277R mutant plasmids were cotransfected with ESCO2-FLAG into NCI-H1975 cells, and acetylation levels were detected using anti-ac-K antibody. We found that the K277R mutant decreased ac-K expression (Fig. 4i). In addition, the K277R mutation influenced the interaction between ESCO2 and hnRNPA1 (Fig. $4 \mathrm{j}$ and $\mathrm{k}$ ). To prove that ESCO2 specifically regulates hnRNPA1 acetylation, recombinant WT hnRNPA1 and its K277R mutant were incubated with recombinant ESCO2, and acetylation levels were detected using anti-ac-K antibody. The acetylation level significantly reduced in the K277R group (Fig. 4l). Collectively, our results indicate that $\mathrm{ESCO} 2$ binds to hnRNPA1 and regulates its acetylation. The acetylation site is at K277, which determines the interaction and acetylation between ESCO2 and hnRNPA1.

\section{ESCO2 acetylates hnRNPA1 at K277 to promote the aggressive phenotype of LUAD cells}

Next, we explore whether ESCO2 promotes the malignant phenotype of LUAD cells by acetylating the K277 site of hnRNP A1.By knocking down the expression of hnRNPA1 by siRNA, the effect of the background expression of hnRNP A1 was eliminated. The hnRNPA1 siRNAs (small interfering RNAs) together with ESCO2 plasmids were transfected into LUAD cells, and hnRNPA1 expression was restored using WT hnRNPA1 and the mutant K277R and K277Q plasmids (Fig. 5a). Compared with the NC group, silencing hnRNPA1 significantly suppressed LUAD cell growth, colony formation, migration, and invasion in the NCI-H1975 and A549 cells (Fig. 5b-d). Furthermore, silencing hnRNPA1 antagonized the enhancement of LUAD cell growth, colony formation, migration, and invasion induced by ESCO2 overexpression, indicating that ESCO2 promotes malignant progression through hnRNPA1 (Fig. 5b-d). Interestingly, when the WT hnRNPA1 vector or hnRNPA1 K277Q mutant restored hnRNPA1 expression in the LUAD cells, ESCO2 significantly promotes the malignant phenotype of LUAD cells, but not in the hnRNPA1 K277R mutant condition, as ESCO2 could not acetylate it (Fig. 5b-d). Collectively, these data show that ESCO2 promotes LUAD cell proliferation and metastasis by acetylating the oncoprotein hnRNPA1 at K277.

\section{ESCO2 increases hnRNPA1 binding to the intronic sequences flanking exon 9 (EI9) of PKM mRNA by inhibiting hnRNPA1 nuclear export}

The K277 site of hnRNPA1 is located in the M9 domain, which mediates hnRNPA1 nuclear transport $[28,29]$. Therefore, we investigated if hnRNPA1 acetylation by ESCO2 affects its nuclear localization. The ESCO2FLAGplasmid was transfected into NCI-H1975 cells; the nucleus and cytoplasm were separated. hnRNPA1 expression in the ESCO2 overexpression cells was upregulated in the nucleus, and was downregulated in the cytosol (Fig. 6a). Immunofluorescence staining indicated that ESCO2 overexpression retained hnRNPA1 in the nucleus (Fig. 6b). ESCO2 overexpression upregulated PKM2 protein expression levels and downregulated 


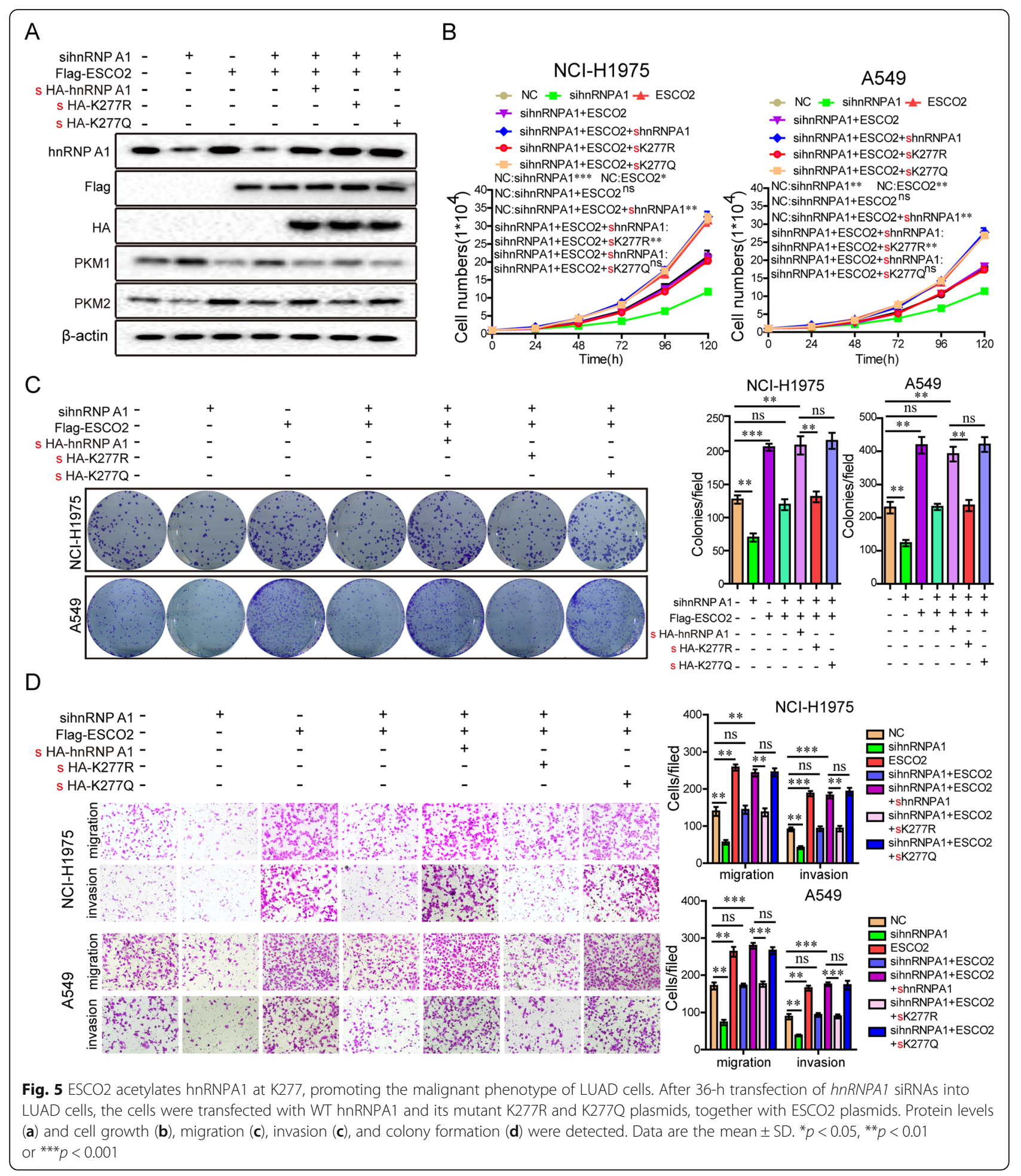

PKM1 protein expression levels, but did not change hnRNPA1 expression levels (Fig. 6c).

hnRNPA1 restricts the inclusion of PKM exon 9, promoting PKM2 formation and inhibiting PKM1 formation, by binding to the UAGGGC sequences of exon 9 $[23,30]$. RNA pull-down experiments showed that
hnRNPA1 can strongly bind to the PKM EI9 (50-68) sequence, and when the G3 nucleotide of EI9 (50-68) is mutated to $\mathrm{C}$, its ability to bind to hnRNPA1 is significantly reduced (Fig. 6d). At the same time, ESCO2 did not directly bind to the EI9 (50-68) sequence (Fig. 6d). Moreover, ESCO2 increased hnRNPA1 and PKM EI9 


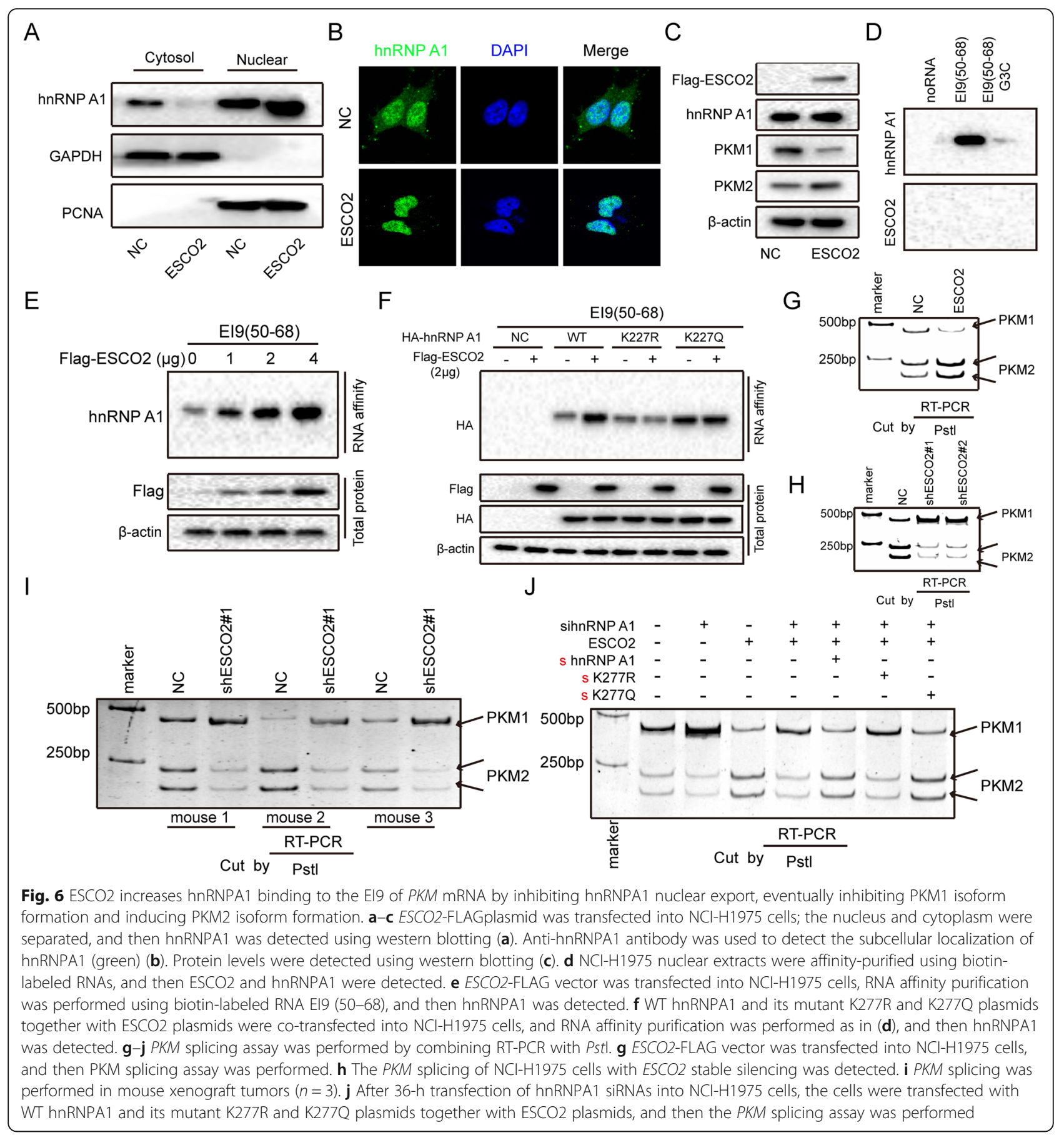

(50-68) binding in a dose-dependent manner in the nucleus (Fig. 6e). To investigate whether ESCO2 increases hnRNPA1 to PKM EI9 (50-68) binding by acetylating hnRNPA1, we transfected hnRNPA1 siRNAs into NCIH1975 cells for $36 \mathrm{~h}$, and then co-transfected the cells with WT hnRNPA1 and its mutant K277R and K277Q plasmids together with ESCO2 plasmids RNA pull-down using biotin-labeled EI9 (50-68) RNA showed that that ESCO2 promoted the binding of WT hnRNPA1 to PKM
EI9 (50-68), but not that of the K277R mutant. In addition, compared to the WT hnRNPA1, the K277Q mutant had higher affinity with PKM EI9 (50-68), and ESCO2 overexpression did not increase hnRNPA1 binding to PKM EI9 (50-68) (Fig. 6f). Next, we used RTPCR and Pst restriction digestion to investigate whether ESCO2 regulates alternative splicing of $P K M$ premRNA. ESCO2 overexpression decreased PKM1 isoform mRNA levels and increased that of the PKM2 isoform 
(Fig. 6g). Silencing ESCO2 decreased PKM2 isoform mRNA levels and increased that of the PKM1 isoform (Fig. 6h); the same results were obtained for PKM splicing in the mouse xenograft tumors $(n=3)$ (Fig. 6i). Compared with the NC group, silencing hnRNPA1 suppressed $P K M 2$ isoform mRNA levels and increased that of the PKM1 isoform, and silencing hnRNPA1 antagonized the splicing change of $P K M$ pre-mRNA induced by $\mathrm{ESCO} 2$ overexpression (Fig. 6j). Furthermore, when the WT hnRNPA1 vector or hnRNPA1 K277Q mutant restored hnRNPA1 expression in the LUAD cells, ESCO2 could decrease PKM1 isoform mRNA levels and increased that of the PKM2 isoform, but not in the hnRNPA1 K277R mutant condition. Collectively, these data show that ESCO2 increases hnRNPA1 binding to the EI9 of PKM mRNA by inhibiting hnRNPA1 nuclear translocation, eventually inhibiting PKM1 isoform formation and inducing PKM2 isoform formation.

\section{ESCO2 promotes aerobic glycolysis of LUAD cells by increasing PKM2 expression and decreasing PKM1 expression}

The PKM2 isozyme is a key promoter of the Warburg effect in tumors, which is characterized by increased glucose uptake and lactic acid production [31]. PKM protein levels were detected in NCI-H1975 cells with ESCO2 stable silencing, and showed that PKM1 expression was obviously increased, while PKM2 expression was obviously decreased (Fig. 7a). Silencing ESCO2 significantly decreased glucose uptake and lactate production in the LUAD cells (Fig. 7b and c). In addition, ESCO2 overexpression significantly increased glucose uptake and lactate production in the LUAD cells (Fig. S2). Compared with the NC group, silencing hnRNPA1 also significantly decreased glucose uptake and lactate production, and antagonized the glucose uptake and lactate production promoted by ESCO2 overexpression (Fig. 7d and e). Furthermore, when the WT hnRNPA1 vector or hnRNPA1 K277Q mutant restored hnRNPA1 expression in the LUAD cells, ESCO2 significantly promotes glucose uptake and lactate production, but not in the hnRNPA1 K277R mutant condition (Fig. 7d and e). The working model of ESCO2 promoted aerobic glycolysis and drove malignant progression by acetylating hnRNPA1 (Fig. 7f).

\section{Discussion}

In the present study, literature and database analyses showed that ESCO2 has a significant correlation with malignant progression of LUAD. Here, comparison of tumor-adjacent normal lung tissue via analysis of the Oncomine, GEO and TCGA datasets showed that ESCO2 mRNA and protein expression levels were upregulated in LUAD tissues. GSEA showed that the cell cycle signatures and DNA replication signatures had a significant positive correlation with ESCO2 mRNA expression levels in TCGA LUAD cohort. Our results show that The ESCO2 overexpression NCI-H1975 and A549 cells had greater cell growth and greater invasive, migration, and colony-formation ability, while silencing ESCO2 had the opposite effect. Furthermore, ESCO2 stable silencing decreased the tumor growth and pulmonary metastasis of the NCI-H1975 cells in vivo. Chen et al. discovered that ESCO2 knockdown dramatically inhibited cell proliferation and induced apoptosis in human gastric cancer cells, and suppressed tumor xenograft development in vivo [19]. In addition, significantly high ESCO2 expression was found in renal cell carcinoma tissue and cell lines, promoting cell aggressive behaviors and inducing poor prognosis [15]. Therefore, together with other research data, our data indicate that ESCO2 plays a key role in the proliferation and metastasis of many types of human cancer.

Furthermore, we found that ESCO2 could interact with hnRNPA1 and acetylates hnRNPA1 at K277. hnRNPA1 regulates alternative splicing of interferon regulatory factor 3 and affects immunomodulatory functions in human non-small cell lung cancer cells [32]. hnRNPA1 plays a crucial role in regulating cell proliferation, invasiveness, metabolism, and immortalization in multiple tumors such as hepatocellular carcinoma, prostate cancer, and oral squamous cell cancer [33-35]. In the present study, silencing hnRNPA1 antagonized the enhancement of LUAD cell growth, colony formation, migration, and invasion induced byESCO2 overexpression. The data suggest that the ESCO2 and hnRNPA1 interaction promotes the malignant progression of LUAD.

Here, we discovered a novel acetylated substrate, hnRNPA1, of the acetyltransferase ESCO2. Furthermore, we found that ESCO2 acetylates hnRNPA1 at K277 and inhibits the nuclear export of hnRNPA1. In addition, hnRNPA1 mediated the regulation of $P K M$ splicing by blocking the binding of the arginine residues in the RGG motif of hnRNPA1 to the PKM EI9, ensuring the formation of PKM2 and suppressing glucose metabolism reprogramming [26]. We discovered that ESCO2 increased hnRNPA1 binding to the EI9 of PKM mRNA by inhibiting hnRNPA1 nuclear translocation, leading to increased PKM2 expression and decreased PKM1 expression.

We also elucidated the function and mechanism of ESCO2 in glucose metabolism in LUAD cells. ESCO2 promoted aerobic glycolysis of LUAD cells by increasing PKM2 expression and decreasing PKM1 expression. PKM is a glycolytic enzyme that catalyzes the final step in glycolysis, and exists in two different forms: PKM1 and PKM2. PKM1 is distributed in high energy-demand organs, such as brain and muscle. PKM2 is believed to 


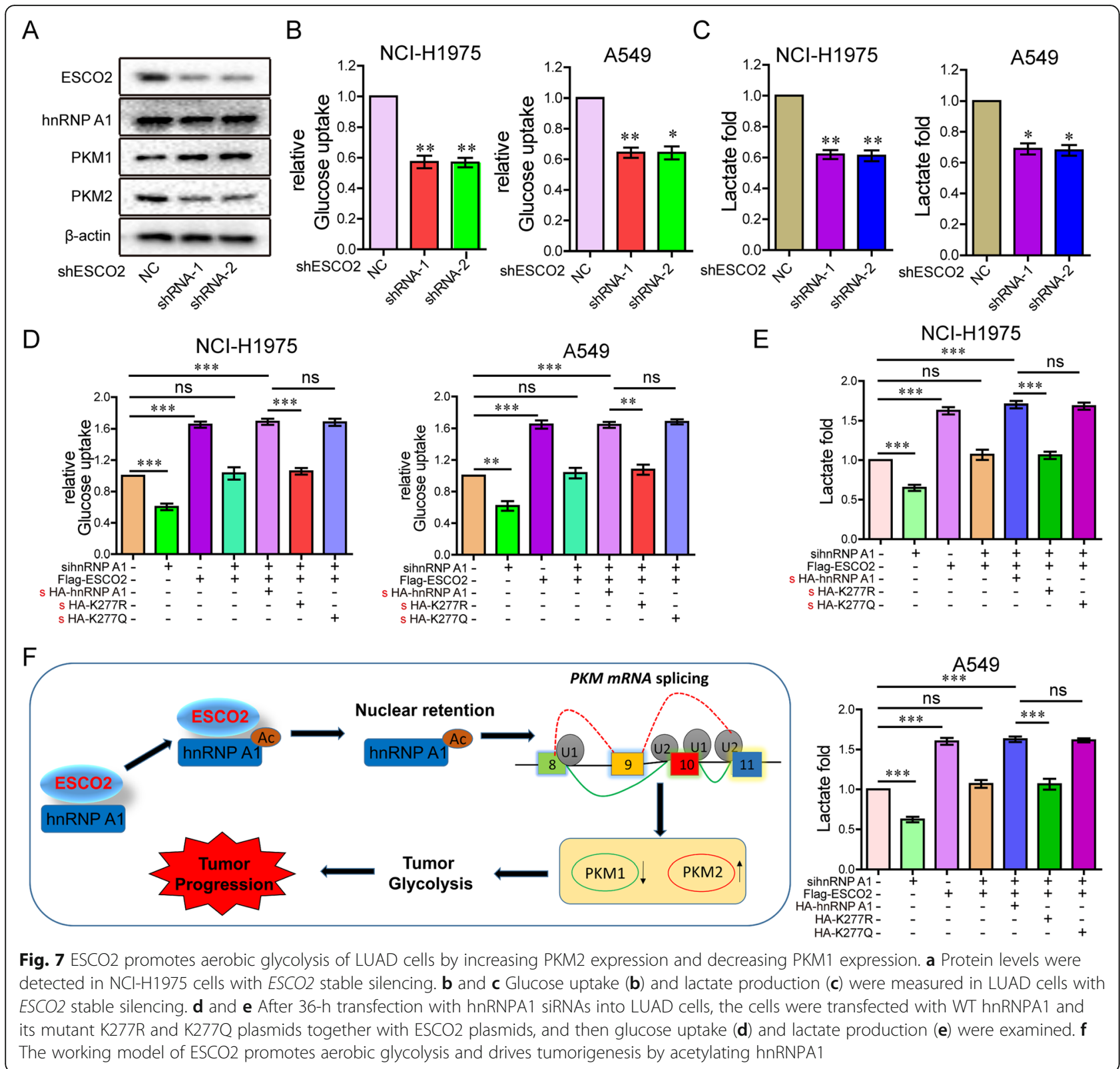

be one of the most important genes in cancer-specific energy metabolism, known as the Warburg effect [36]. Most cancer cells such as that of colon cancer, bladder cancer, and pancreatic cancer express PKM2 dominantly to maintain a glycolysis-dominant energy metabolism [24, 37-39]. PKM2 reduces the glucose levels for intracellular utilization, in particular citrate production, thereby increasing the $\alpha$-ketoglutarate/citrate ratio to promote the generation of glutamine-derived acetylcoenzyme A through the reductive pathway. In addition, reductive glutamine metabolism promotes cell proliferation under hypoxia conditions and supports in vivo tumor growth [40]. Therefore, we have found and proven that ESCO2 is an important functional molecule that promotes metabolic reprogramming of LUAD. The ESCO2-cohesin complex links DNA molecules and plays important roles in the gene transcription of eukaryotic genomes. Sadia Rahman and their colleagues have found that Esco2 binding sites are enriched for CTCF and REST/NRSF transcription factor motifs [41]. In addition, ESCO2 can regulate transcription of neuron-specific genes in other tissues [42, 43]. These studies show that ESCO2 is closely related to RNA transcription regulation. Whether ESCO2 promotes the malignant progression of lung adenocarcinoma by regulating gene transcription and its specific regulatory mechanisms, this will be the focus of our further research. 


\section{Conclusion}

In conclusion, patients with LUAD with high ESCO2 mRNA and protein expression levels have lower OS and recurrence-free survival, as compared with patients with low ESCO2 expression levels. A novel role for ESCO2 in LUAD tumorigenesis has been elucidated, that is, $E S C O 2$ upregulation promotes cell growth, proliferation, colony formation, and cell cycle progression. Moreover, ESCO2 can interact with hnRNPA1 and acetylates it at K277, retaining it in the nucleus. In addition, ESCO2 promotes hnRNPA1 binding to the EI9 of PKM mRNA by inhibiting hnRNPA1 nuclear translocation. Furthermore, ESCO2 promotes aerobic glycolysis of LUAD cells by increasing PKM2 expression and decreasing PKM1 expression. Therefore, ESCO2 may serve as a new therapeutic target for LUAD.

\section{Supplementary Information}

The online version contains supplementary material available at https:/doi. org/10.1186/s13046-021-01858-1.

Additional file 1: Supplementary Figure 1. (A) hnRNPA1-HA vector was transfected into HEK293T cells, the hnRNPA1-HA complexes underwent Co-IP, and then protein modification of hnRNPA1 was identified by Coomassie blue staining with MS.

Additional file 2: Supplementary Figure 2. (A-B) ESCO2-FLAG vector was transfected into $\mathrm{NCl}-\mathrm{H} 1975$ cells, and then Glucose uptake (A) and lactate production (B) were measured.DD.

Additional file 3: Supplementary Table S1. The antibodies, primers, oligonucleotides and shRNAs used in this study are shown.

Additional file 4: Supplementary Table S2.

\section{Abbreviations}

ESCO2: Establishment of cohesion 1 homolog 2; hnRNPA1: Heterogeneous nuclear ribonucleoprotein A1; LUAD: Lung adenocarcinoma; CoIP: Coimmunoprecipitation; MS: Mass spectrometry; GSEA: Gene set enrichment analysis

\section{Acknowledgments}

Not applicable.

\section{Authors' contributions}

HC and WPC conceived and designed the experiments. HEZ, and WPC analyzed the data, HEZand SNS prepared the manuscript. HEZ, TL andSNS performed the experiments. $\mathrm{HC}$ and DXC provided statistical support and analyzed the IHC data. All authors read and approved the final manuscript.

\section{Funding}

The study was supported by the Third Affiliated Hospital of Guangzhou Medical University Youth fund (grant no.2018Q19 and 2019Q2).

\section{Availability of data and materials}

All data generated or analyzed during this study are included in thispublished article.

\section{Ethics approval and consent to participate}

This study was approved by the Ethical Committee of the thirdAffiliated Hospital of Guangzhou Medical University.

\section{Consent for publication}

Not applicable.

\section{Competing interests}

The authors declare no conflicts of interest.

\section{Author details}

${ }^{1}$ Department of General Practice, The Third Affiliated Hospital of Guangzhou Medical University, Guangzhou Medical University, Guangzhou, Guangdong 510150, P.R. China. ${ }^{2}$ Department of Anesthesiology, The Third Affiliated Hospital of Guangzhou Medical University, Guangzhou Medical University, Guangzhou, Guangdong 510150, P.R. China. ${ }^{3}$ State Key Laboratory of Animal Breeding, Institute of Animal Science, Guangdong Academy of Agricultural Sciences, South China Key Laboratory of Animal Nutrition and Feed, Ministry of Agriculture, Guangzhou 510640, P. R. China. ${ }^{4}$ Department of Respiratory, The People's Hospital of Qingyuan, Sixth Affiliate Hospital of Guangzhou Medical University, Qingyuan 511518, P. R. China. ${ }^{5}$ Department of Pathology, The Third Affiliated Hospital of Guangzhou Medical University, Guangzhou Medical University, Guangzhou, Guangdong 510150, P.R. China.

Received: 19 October 2020 Accepted: 25 January 2021

Published online: 11 February 2021

\section{References}

1. Lambe G, Durand M, Buckley A, Nicholson S, McDermott R. Adenocarcinoma of the lung: from BAC to the future. Insights Imaging. 2020;11(1):69.

2. $\quad$ Liang W, Zhang L, Jiang G, Wang Q, Liu L, Liu D, et al. Development and validation of a nomogram for predicting survival in patients with resected non-small-cell lung cancer. J Clin Oncol. 2015;33(8):861-9.

3. Chen W, Zheng R, Baade PD, Zhang S, Zeng H, Bray F, et al. Cancer statistics in China, 2015. CA Cancer J Clin. 2016;66(2):115-32.

4. Zhu HE, Yin JY, Chen DX, He S, Chen H. Agmatinase promotes the lung adenocarcinoma tumorigenesis by activating the NO-MAPKs-PI3K/Akt pathway. Cell Death Dis. 2019;10(11):854.

5. Shaw AT, Solomon BJ, Besse B, Bauer TM, Lin CC, Soo RA, et al. ALK resistance mutations and efficacy of Lorlatinib in advanced anaplastic lymphoma kinase-positive non-small-cell lung Cancer. J Clin Oncol. 2019; 37(16):1370-9

6. Kobayashi K, Soejima K, Fukunaga K, Shintani Y, Sekine I, Shukuya T, et al. Key prognostic factors for EGFR-mutated non-adenocarcinoma lung cancer patients in the Japanese joint Committee of Lung Cancer Registry Database. Lung Cancer. 2020;146:236-43.

7. Chen JA, Riess JW. Optimal Management of Patients with advanced NSCLC harboring high PD-L1 expression and driver mutations. Curr Treat Options in Oncol. 2020;21(7):60.

8. Sankar K, Gadgeel SM, Qin A. Molecular therapeutic targets in non-small cell lung cancer. Expert Rev Anticancer Ther. 2020;20(8):647-61.

9. Noor ZS, Cummings AL, Johnson MM, Spiegel ML, Goldman JW. Targeted therapy for non-small cell lung Cancer. Semin Respir Crit Care Med. 2020; 41(3):409-34.

10. Gu J, Yao W, Shi P, Zhang G, Owonikoko TK, Ramalingam SS, et al. MEK or ERK inhibition effectively abrogates emergence of acquired osimertinib resistance in the treatment of epidermal growth factor receptor-mutant lung cancers. Cancer. 2020;126(16):3788-99.

11. Calvayrac O, Pradines A, Pons E, Mazieres J, Guibert N. Molecular biomarkers for lung adenocarcinoma. Eur Respir J. 2017:49(4):1601734.

12. Testa U, Castelli G, Pelosi E. Lung Cancers: Molecular Characterization, Clonal Heterogeneity and Evolution, and Cancer Stem Cells. Cancers (Basel). 2018; 10(8):248

13. Alomer RM, da Silva EML, Chen J, Piekarz KM, McDonald K, Sansam CG, et al. Esco1 and Esco2 regulate distinct cohesin functions during cell cycle progression. Proc Natl Acad Sci U S A. 2017;114(37):9906-11.

14. Guo XB, Huang B, Pan YH, Su SG, Li Y. ESCO2 inhibits tumor metastasis via transcriptionally repressing MMP2 in colorectal cancer. Cancer Manag Res. 2018;10:6157-66.

15. Wang QL, Liu L. Establishment of cohesion 1 homolog 2 facilitates cell aggressive behaviors and induces poor prognosis in renal cell carcinoma. J Clin Lab Anal. 2020;34(5):e23163.

16. Xiao B, Chen L, Ke Y, Hang J, Cao L, Zhang R, et al. Identification of methylation sites and signature genes with prognostic value for luminal breast cancer. BMC Cancer. 2018;18(1):405. 
17. Zhang W, Cui Q, Qu W, Ding X, Jiang D, Liu H. TRIM58/cg26157385 methylation is associated with eight prognostic genes in lung squamous cell carcinoma. Oncol Rep. 2018;40(1):206-16.

18. Ryu B, Kim DS, Deluca AM, Alani RM. Comprehensive expression profiling of tumor cell lines identifies molecular signatures of melanoma progression. PLoS One. 2007:2(7):e594.

19. Chen H, Zhang L, He W, Liu T, Zhao Y, Chen H, et al. ESCO2 knockdown inhibits cell proliferation and induces apoptosis in human gastric cancer cells. Biochem Biophys Res Commun. 2018;496(2):475-81.

20. Kedzierska H, Piekielko-Witkowska A. Splicing factors of SR and hnRNP families as regulators of apoptosis in cancer. Cancer Lett. 2017;396:53-65.

21. Kutluay SB, Emery A, Penumutchu SR, Townsend D, Tenneti K, Madison MK, et al. Genome-Wide Analysis of Heterogeneous Nuclear Ribonucleoprotein (hnRNP) Binding to HIV-1 RNA Reveals a Key Role for hnRNP H1 in Alternative Viral mRNA Splicing. J Virol. 2019:93(21):e01048-19.

22. Luo W, Semenza GL. Emerging roles of PKM2 in cell metabolism and cancer progression. Trends Endocrinol Metab. 2012;23(11):560-6.

23. David CJ, Chen M, Assanah M, Canoll P, Manley JL. HnRNP proteins controlled by c-Myc deregulate pyruvate kinase mRNA splicing in cancer. Nature. 2010;463(7279):364-8.

24. Kuranaga Y, Sugito $N$, Shinohara $H$, Tsujino T, Taniguchi K, Komura K, et al. SRSF3, a Splicer of the PKM Gene, Regulates Cell Growth and Maintenance of Cancer-Specific Energy Metabolism in Colon Cancer Cells. Int J Mol Sci. 2018;19(10):3012.

25. Robbins Y, Greene S, Friedman J, Clavijo PE, Van Waes C, Fabian KP, et al. Tumor control via targeting PD-L1 with chimeric antigen receptor modified NK cells. Elife. 2020;9:e54854.

26. Huang JZ, Chen M, Chen D, Gao XC, Zhu S, Huang H, et al. A peptide encoded by a putative IncRNA HOXB-AS3 suppresses Colon Cancer growth. Mol Cell. 2017;68(1):171-84 e6.

27. Chabot B, LeBel C, Hutchison S, Nasim FH, Simard MJ. Heterogeneous nuclear ribonucleoprotein particle $\mathrm{a} / \mathrm{B}$ proteins and the control of alternative splicing of the mammalian heterogeneous nuclear ribonucleoprotein particle A1 premRNA. Prog Mol Subcell Biol. 2003;31:59-88.

28. Izaurralde E, Jarmolowski A, Beisel C, Mattaj IW, Dreyfuss G, Fischer U. A role for the M9 transport signal of hnRNP A1 in mRNA nuclear export. J Cell Biol. 1997;137(1):27-35.

29. lijima M, Suzuki M, Tanabe A, Nishimura A, Yamada M. Two motifs essential for nuclear import of the hnRNP A1 nucleocytoplasmic shuttling sequence M9 core. FEBS Lett. 2006;580(5):1365-70.

30. Chen M, David CJ, Manley JL. Concentration-dependent control of pyruvate kinase M mutually exclusive splicing by hnRNP proteins. Nat Struct Mol Biol. 2012;19(3):346-54

31. Chen M, Sheng XJ, Qin YY, Zhu S, Wu QX, Jia L, et al. TBC1D8 amplification drives tumorigenesis through metabolism reprogramming in ovarian Cancer. Theranostics. 2019;9(3):676-90.

32. Guo R, Li Y, Ning J, Sun D, Lin L, Liu X. HnRNP A1/A2 and SF2/ASF regulate alternative splicing of interferon regulatory factor-3 and affect immunomodulatory functions in human non-small cell lung cancer cells. PLoS One. 2013;8(4):e62729.

33. Yang H, Zhu R, Zhao X, Liu L, Zhou Z, Zhao L, et al. Sirtuin-mediated deacetylation of hnRNP A1 suppresses glycolysis and growth in hepatocellular carcinoma. Oncogene. 2019;38(25):4915-31.

34. Carabet LA, Leblanc E, Lallous N, Morin H, Ghaidi F, Lee J, et al. ComputerAided Discovery of Small Molecules Targeting the RNA Splicing Activity of hnRNP A1 in Castration-Resistant Prostate Cancer. Molecules. 2019;24(4):763.

35. Yu C, Guo J, Liu Y, Jia J, Jia R, Fan M. Oral squamous cancer cell exploits hnRNP A1 to regulate cell cycle and proliferation. J Cell Physiol. 2015;230(9): 2252-61.

36. Taniguchi K, Sugito N, Shinohara H, Kuranaga Y, Inomata Y, Komura K, et al. Organ-Specific MicroRNAs (MIR122, 137, and 206) Contribute to Tissue Characteristics and Carcinogenesis by Regulating Pyruvate Kinase M1/2 (PKM) Expression. Int J Mol Sci. 2018;19(5):1276.

37. Massari F, Ciccarese C, Santoni M, lacovelli R, Mazzucchelli R, Piva F, et al, Metabolic phenotype of bladder cancer. Cancer Treat Rev. 2016;45:46-57.

38. Calabretta S, Bielli P, Passacantilli I, Pilozzi E, Fendrich V, Capurso G, et al. Modulation of PKM alternative splicing by PTBP1 promotes gemcitabine resistance in pancreatic cancer cells. Oncogene. 2016;35(16):2031-9.

39. Zhao $X$, Zhu Y, Hu J, Jiang L, Li L, Jia S, et al. Shikonin inhibits tumor growth in mice by suppressing pyruvate kinase M2-mediated aerobic glycolysis. Sci Rep. 2018;8(1):14517.
40. Liu M, Wang Y, Ruan Y, Bai C, Qiu L, Cui Y, et al. PKM2 promotes reductive glutamine metabolism. Cancer Biol Med. 2018;15(4):389-99.

41. Rahman S, Jones MJ, Jallepalli PV. Cohesin recruits the Esco1 acetyltransferase genome wide to repress transcription and promote cohesion in somatic cells. Proc Natl Acad Sci U S A. 2015;112(36):11270-5.

42. Ooi L, Wood IC. Chromatin crosstalk in development and disease: lessons from REST. Nat Rev Genet. 2007;8(7):544-54.

43. Qureshi IA, Gokhan S, Mehler MF. REST and CoREST are transcriptional and epigenetic regulators of seminal neural fate decisions. Cell Cycle. 2010;9(22): 4477-86.

\section{Publisher's Note}

Springer Nature remains neutral with regard to jurisdictional claims in published maps and institutional affiliations.
Ready to submit your research? Choose BMC and benefit from:

- fast, convenient online submission

- thorough peer review by experienced researchers in your field

- rapid publication on acceptance

- support for research data, including large and complex data types

- gold Open Access which fosters wider collaboration and increased citations

- maximum visibility for your research: over $100 \mathrm{M}$ website views per year

At BMC, research is always in progress.

Learn more biomedcentral.com/submissions 